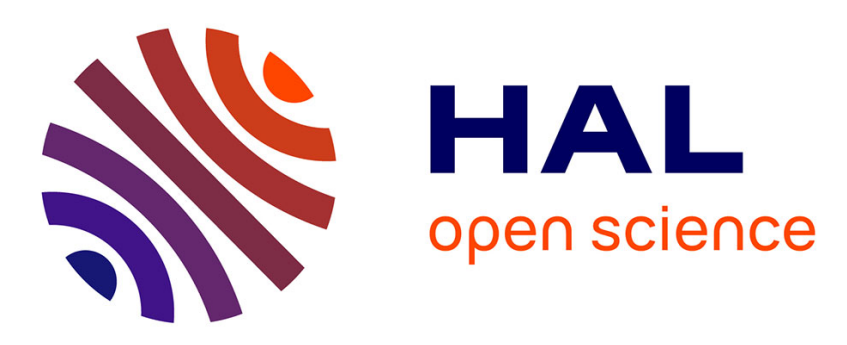

\title{
System-level performance analysis of relay-aided multiple-antenna cellular networks
}

\author{
Konstantinos Ntontin, Marco Di Renzo, Christos Verikoukis
}

\section{To cite this version:}

Konstantinos Ntontin, Marco Di Renzo, Christos Verikoukis. System-level performance analysis of relay-aided multiple-antenna cellular networks. 2016 IEEE 27th Annual International Symposium on Personal, Indoor, and Mobile Radio Communications (PIMRC), Sep 2016, Valencia, Spain. 10.1109/PIMRC.2016.7794656 . hal-01880159

\section{HAL Id: hal-01880159 \\ https://hal.science/hal-01880159}

Submitted on 16 Jul 2020

HAL is a multi-disciplinary open access archive for the deposit and dissemination of scientific research documents, whether they are published or not. The documents may come from teaching and research institutions in France or abroad, or from public or private research centers.
L'archive ouverte pluridisciplinaire HAL, est destinée au dépôt et à la diffusion de documents scientifiques de niveau recherche, publiés ou non, émanant des établissements d'enseignement et de recherche français ou étrangers, des laboratoires publics ou privés. 


\title{
System-Level Performance Analysis of Relay-Aided Multiple-Antenna Cellular Networks
}

\author{
Konstantinos Ntontin ${ }^{(1,2)}$, Marco Di Renzo ${ }^{(3)}$, and Christos Verikoukis ${ }^{(4)}$ \\ ${ }^{1}$ Electronics Department, University of Barcelona, Spain \\ ${ }^{2}$ European Projects Department, SingularLogic, S.A., Greece \\ ${ }^{(3)}$ L2S, UMR 8506 CNRS - SUPELEC - Univ Paris-Sud, Laboratory of Signals and Systems (L2S) \\ French National Center for Scientific Research (CNRS), École Supérieure d'Électricité (SUPÉLEC) \\ University of Paris-Sud XI (UPS), 3 rue Joliot-Curie, 91192 Gif-sur-Yvette (Paris), France \\ ${ }^{(4)}$ Telecommunications Technological Centre of Catalonia (CTTC) \\ Castelldefels, Spain \\ Email: konstantinos.ntontin.1983@iee.org, marco.direnzo@1ss.supelec.fr, cveri@cttc.es
}

\begin{abstract}
Motivated by recent results regarding the performance analysis, in terms of coverage probability and average rate, of microwave single-antenna cellular networks with HalfDuplex (HD) Relay Nodes (RNs) by using a stochastic-geometry approach, in this work we extend this study by considering multiple-antenna Base Stations (BSs) and RNs. Analytical formulas are provided for the achieved coverage probability and average rate of these networks, which are shown to closely match the simulation results. In addition, the simulation results show that the availability or not of a large antenna array determines whether RNs can be beneficial to the network in terms of coverage improvement. In particular, substantial coverage gains are observed for a massive-antenna deployment, whereas no rate gains are achieved due to the half-duplex constraint of the RNs.
\end{abstract}

\section{INTRODUCTION}

The deployment of RNs is considered to be a viable option, mainly for the coverage improvement of conventional cellular networks, especially at the cell-edge regions. In particular, it has been included in modern standards, such as the IEEE 802.16j working group [1] and the Third Generation Partnership Project's Long Term Evolution Advanced (3GPP LTE-A) [2]. It has also been studied in various literature works, which despite the fact that they show that relaying can be beneficial regarding coverage and rate improvement with respect to the no-relay case, they consider scenarios that are difficult to be realized in practice, such as Base Stations (BSs) and Relay Nodes (RNs) located at fixed positions [3], [4] and interference that is Gaussian distributed [5], [6].

Against this background and to the best of the author's knowledge, [7] is the first work that studies relay-aided cellular networks by considering a stochastic geometry abstraction modeling, where the BSs, RNs and Mobile Terminals (MTs) are modeled as three independent and homogeneous Poisson Point Processes (PPPs) [8]. Stochastic geometry as a tool has been widely used in the recent years for the systemlevel performance analysis of cellular networks due to its analytical tractability and the relatively small gap that the analysis provides with respect to real-world measurements [9], [10]. By employing this tool, the main outcomes in [7] are that coverage and rate highly depend on the path-loss exponents of one- and two-hop links and that parameter optimization is needed to achieve gains by using relays in certain scenarios. However, [7] considers only single-antenna nodes.

Contribution: In this paper, we extend the analysis of [7] by considering multiple-antenna $\mathrm{BSs}$ and $\mathrm{RNs}$, which can beamform their signal towards their intended receiver. We derive analytical expressions of the coverage probability and average rate, which exhibit a close match with respect to Monte Carlo simulations. In addition, the results show that while substantial coverage-probability gains are achieved if a large antenna array is available at the BSs and the RNs, a rate reduction is observed if the aim of the parameter optimization is the maximization of the coverage probability, which is attributed to the half-duplex constraint of the RNs.

Organization: The rest of the paper is organized as follows: In Section II, the system model is presented. Section III introduces the signal model and the examined performance metrics. In Section IV, we derive analytical expressions of the coverage probability and average rate, which are validated in Section V by means of Monte Carlo simulations. Finally, Section VI concludes this work.

\section{SySTEM MODEL}

\section{A. Scenario}

We consider a downlink relay-aided cellular network, where the BSs, RNs, and MTs are modeled as points of three independent and homogeneous Poisson Point Processes (PPPs), which are denoted by $\Phi_{\mathrm{BS}}, \Phi_{\mathrm{RN}}$, and $\Phi_{\mathrm{MT}}$ with densities $\lambda_{\mathrm{BS}}, \lambda_{\mathrm{RN}}$, and $\lambda_{\mathrm{MT}}$, respectively. BSs are equipped with $N_{B S}$ antennas, RNs use a single antenna for reception, $N_{R}$ antennas for transmission ${ }^{1}$, and act in the Half-Duplex (HD) mode and, finally, MTs are single-antenna nodes. In addition, we assume that they have perfect instantaneous Channel State Information (CSI) regarding their intended receiver only. The BSs and RNs have $N_{R B}$ Resource Blocks (RBs) at their disposal, which

\footnotetext{
${ }^{1}$ This means that RNs have separate receiver and transmitter units, which are connected to a single and $N_{R S}$ antennas, respectively.
} 
serve the MTs. Furthermore, we assume that the BSs pick randomly with probability $1 / N_{R B}$ the $\mathrm{RB}$ to transmit to a particular MT. In addition, the RNs transmit at the same RB with the BSs they are paired with after the cell association is performed. Regarding the relaying protocol, we assume that the end-to end communication takes place in two time slots, where during the first time slot only the BSs are allowed to transmit while at the second time slot only the RNs can transmit.

Moreover, we denote a particular $\mathrm{MT}$ as $\mathrm{MT}_{0}$, which can be served via a one or a two-hop transmission. In the former case, the serving $\mathrm{BS}$ is denoted by $\mathrm{BS}_{0}$, whereas in the latter one the $\mathrm{RN}$ that serves $\mathrm{MT}_{0}$ is denoted by $\mathrm{RN}_{0}$ and the $\mathrm{BS}$ that serves $\mathrm{RN}_{0}$ is denoted by $\mathrm{BS}_{\mathrm{R} 0}$. Without loss of generality and based on the Slivnyak theorem [8], in this work we study the performance metrics of a $\mathrm{MT}_{0}$ located at the origin of the bi-dimensional plane. Finally, we note that the analysis is performed for an arbitrary RB and the set of interfering BSs and RNs in the RB of interest are denoted by $\Phi_{\mathrm{BS}}^{(\mathrm{I})}$ and $\Phi_{\mathrm{RN}}^{(\mathrm{I})}$, respectively.

\section{B. Channel Modeling}

1) Path-Loss Model: Let $r_{X_{i}, Y_{k}}$ be the distance between the nodes $X_{i}$ and $Y_{k}$ with path $\operatorname{loss} l\left(r_{X_{i}, Y_{k}}\right)=\kappa_{0} r_{X_{i}, Y_{k}}^{\beta_{X}}$, where $\kappa_{0}$ denotes the free-space path loss at a distance of one meter and $\beta_{X, Y}>2$ denotes the path-loss exponent. Specifically, $\kappa_{0}=(4 \pi / \nu)^{2}$, where $\nu$ is the transmission wavelength.

2) Shadowing Model: We assume that the link between the nodes $X_{i}$ and $Y_{k}$ is subject to shadow fading, which follows a log-normal distribution with parameters $\mu_{X, Y}$ and $\sigma_{X, Y}^{2}$.

3) Fast-Fading: Rayleigh fading with unit variance is assumed for the links between any of the antennas of $X_{i}$ and $Y_{k}$.

\section{Cell Association and Relaying Protocol}

A DF relaying protocol is considered [7]. All transmissions in the same RB occur at the same transmission wavelength $\nu$. Let $P_{T}$ be the total transmit power budget for serving $\mathrm{MT}_{0}$. Let $P_{\mathrm{BS}_{0}}, P_{\mathrm{RN}_{0}}$ and $P_{\mathrm{BS}_{\mathrm{R} 0}}$ denote the transmit power budgets of $\mathrm{BS}_{0}, \mathrm{RN}_{0}$ and $\mathrm{BS}_{\mathrm{R} 0}$. In order to ensure the total power constraint, they are defined as $P_{\mathrm{BS}_{0}}=P_{T}, P_{\mathrm{RN}_{0}}=\left(1-K_{T}\right) P_{T}$ and $P_{\mathrm{BS}_{\mathrm{R} 0}}=K_{T} P_{T}$, where $0<K_{T}<1$ is a power splitting coefficient.

The triplet $\mathrm{BS}_{0}, \mathrm{RN}_{0}$ and $\mathrm{BS}_{\mathrm{R} 0}$ is identified by using the cell association criterion as follows:

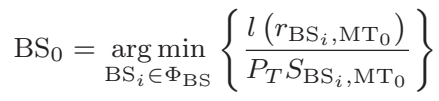

$$
\begin{aligned}
& \mathrm{RN}_{0}=\underset{\mathrm{RN}_{k} \in \Phi_{\mathrm{RN}}}{\arg \min }\left\{\frac{l\left(r_{\mathrm{RN}_{k}, \mathrm{MT}_{0}}\right)}{\left(1-K_{T}\right) P_{T} S_{\mathrm{RN}_{k}, \mathrm{MT}_{0}}}\right\}
\end{aligned}
$$

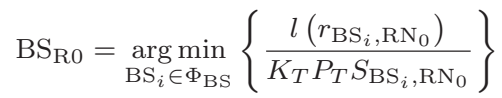

Let the triplet of network elements $\mathrm{BS}_{0}, \mathrm{RN}_{0}$ and $\mathrm{BS}_{\mathrm{R} 0}$ from (1). The typical MT, $\mathrm{MT}_{0}$, is served either via a one- or a two-hop link according to the cell association criterion as follows:

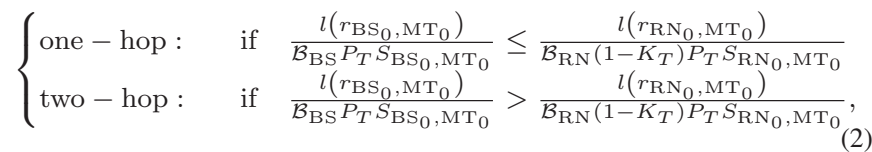

where $\mathcal{B}_{\mathrm{BS}}$ and $\mathcal{B}_{\mathrm{RN}}$ are non-negative bias constants (see [7]), and $\mathrm{BS}_{0}, \mathrm{RN}_{0}, \mathrm{BS}_{\mathrm{R} 0}$ are obtained from (1). Throughout the present paper, without loss of generality, it is assumed that $\mathcal{B}_{\mathrm{RN}}=1$ and $\mathcal{B}_{\mathrm{BS}} \geq 0$. If $\mathcal{B}_{\mathrm{BS}}=1$, there is no bias. If $\mathcal{B}_{\mathrm{BS}} \gg$ 1 , all transmissions are likely to be one-hop. If $\mathcal{B}_{\mathrm{BS}} \ll 1$, all transmissions are likely to be two-hop.

\section{Load Modeling}

Light Traffic Load: In this case, the number of MTs is small. This occurs if $\lambda_{\mathrm{MT}} \ll \mathrm{N}_{\mathrm{RB}} \lambda_{\mathrm{BS}}$ and $\lambda_{\mathrm{MT}} \ll \mathrm{N}_{\mathrm{RB}} \lambda_{\mathrm{RN}}$. Since the RBs are chosen at random and with equal probability by each BS, the density of interfering BSs in a generic RB is equal to $\lambda_{\mathrm{BS}}^{(\mathrm{I})}=\lambda_{\mathrm{MT}} / \mathrm{N}_{\mathrm{RB}}$. As a result, some BSs are inactive in the $\mathrm{RB}$ of interest. The set of interfering BSs in that RB is denoted by $\Phi_{\mathrm{BS}}^{(\mathrm{I})}$ and it can be split in two disjoint sets corresponding to the interfering BSs serving their associated MTs either via a one-, $\Phi_{\mathrm{BS}}^{(\mathrm{I}, 1 \mathrm{hop})}$, or a two-hop, $\Phi_{\mathrm{BS}}^{(\mathrm{I}, 2 \mathrm{hop})}$, link.

Remark 1: Since some BSs are inactive in a generic RB and the cell association in (1), (2) is distance-dependent, the set of interfering BSs in that $\mathrm{RB}, \Phi_{\mathrm{BS}}^{(\mathrm{I})}$, is not a homogeneous PPP. The locations of the interfering BSs are expected to exhibit some level of spatial correlation. For mathematical tractability, we use Approximation 1 for system-level analysis. Its accuracy is studied in Section V.

Approximation 1: The sets of interfering BSs, $\Phi_{\mathrm{BS}}^{(\mathrm{I})}$, $\Phi_{\mathrm{BS}}^{(\mathrm{I}, 1 \text { hop })}$ and $\Phi_{\mathrm{BS}}^{(\mathrm{I}, 2 \mathrm{hop})}$ in a generic RB are assumed to be three homogeneous PPPs of density $\lambda_{\mathrm{BS}}^{(\mathrm{I})}=\lambda_{\mathrm{MT}} / \mathrm{N}_{\mathrm{RB}}$, $\lambda_{\mathrm{BS}}^{(\mathrm{I}, 1 \mathrm{hop})}=\chi_{1 \mathrm{hop}} \lambda_{\mathrm{BS}}^{(\mathrm{I})}$ and $\lambda_{\mathrm{BS}}^{(\mathrm{I}, 2 \mathrm{hop})}=\chi_{2 \mathrm{hop}} \lambda_{\mathrm{BS}}^{(\mathrm{I})}$, respectively. Also, $\Phi_{\mathrm{BS}}^{(\mathrm{I}, 1 \mathrm{hop})}$ and $\Phi_{\mathrm{BS}}^{(\mathrm{I}, 2 \mathrm{hop})}$ are assumed to be independent. The spatial constraints originating from (1) and (2) are, however, taken into account for system-level analysis.

Remark 2: As for the active RNs, comments similar to Remark 1 hold. Hence, $\Phi_{\mathrm{RN}}^{(\mathrm{I})}$ is not a homogeneous PPP. For mathematical tractability, Approximation 2 is used. Its accuracy is studied in Section V.

Approximation 2: The set of interfering RNs, $\Phi_{\mathrm{RN}}^{(\mathrm{I})}$, in a generic RB is assumed to be a homogeneous PPP of density $\lambda_{\mathrm{RN}}^{(\mathrm{I})}=\chi_{2 \mathrm{hop}} \lambda_{\mathrm{BS}}^{(\mathrm{I})}$, where $\lambda_{\mathrm{BS}}^{(\mathrm{I})}=\lambda_{\mathrm{MT}} / \mathrm{N}_{\mathrm{RB}}$. Also, the locations of BSs and RNs are assumed to be independent. Similar to Approximation 1, however, the constraint $\operatorname{card}\left\{\Phi_{\mathrm{RN}}^{(\mathrm{I})}\right\}=\operatorname{card}\left\{\Phi_{\mathrm{BS}}^{(\mathrm{I}, 2 \mathrm{hop})}\right\}$ and the spatial constraints originating from (1) and (2) are taken into account for systemlevel analysis.

Remark 3: In the present paper, due to space limitations, only the light traffic load scenario is investigated, wheras in [7] both the saturated- and light-traffic cases are studied. However, still we get important insights for the system design, as we will see in Section V.

Notation: The following notation is used throughout this paper. i) $s$ is the transmitted data symbol. ii) The random 
variable $\mathcal{Z}_{X, Y}=l\left(r_{X, Y}\right) / S_{X, Y}$ for $X \in\{\mathrm{BS}, \mathrm{RN}\}$ and $Y \in\left\{\mathrm{RN}, \mathrm{MT}_{0}\right\}$ denotes the ratio of path-loss and shadowing for a generic $(X, Y)$ link. iii) $\sigma_{N}^{2}=10^{\sigma_{N}^{2}(\mathrm{dBm}) / 10}$ denotes the thermal noise power, where $\sigma_{N}^{2}(\mathrm{dBm})=-174+$ $10 \log _{10}\left(\mathrm{~B}_{\mathrm{W}}\right)+\mathcal{F}_{\mathrm{dB}}, \mathcal{F}_{\mathrm{dB}}$ is the noise figure in $\mathrm{dB}$ and $\mathrm{B}_{\mathrm{W}}$ is the transmission bandwidth of each RB. iv) $\eta_{\text {cell }}=$ $\left(\mathcal{B}_{\mathrm{BS}} P_{T}\right) /\left(\mathcal{B}_{\mathrm{RN}}\left(1-K_{T}\right) P_{T}\right)$ is a shorthand used for simplifying the writing of (2).

\section{Signal Model And Examined Metrics}

In this section, expressions of the instantaneous signalto-interference plus noise ratio (SINR) are derived and the performance metrics under consideration are presented, which depend on the SINR statistics.

\section{A. Signal Model}

1) Single-Hop Transmission: In the case of a single-hop transmission between $\mathrm{MT}_{0}$ and the selected $\mathrm{BS}, \mathrm{BS}_{0}$, the received signal at $\mathrm{MT}_{0}$, which is denoted by $y_{\mathrm{MT}_{0}}^{(1)}$, is given by

$$
\begin{aligned}
y_{\mathrm{MT}_{0}}^{(1)} & =\sqrt{\left(P_{T} / N_{B S}\right) \mathcal{Z}_{\mathrm{BS}_{0}, \mathrm{MT}_{0}}^{-1}} \mathbf{h}_{\mathrm{BS}_{0}, \mathrm{MT}_{0}} \mathbf{w}_{\mathrm{BS}_{0}, \mathrm{MT}_{0}} s \\
& +i_{\mathrm{BS}, \mathrm{MT}_{0}}^{(1)}+i_{\mathrm{BS}, \mathrm{MT}_{0}}^{(2)}+n_{\mathrm{MT}_{0}}^{(1)},
\end{aligned}
$$

where $\mathbf{h}_{\mathrm{BS}_{0}, \mathrm{MT}_{0}} \in \mathbb{C N}^{1 \times N_{B S}}, \mathbf{w}_{\mathrm{BS}_{0}, \mathrm{MT}_{0}} \in \mathbb{C}^{N_{B S} \times 1}$ is the employed beamforming vector at $\mathrm{BS}_{0}, E\left\{n_{\mathrm{MT}_{0}}^{(1)} n_{\mathrm{MT}_{0}}^{(1) *}\right\}=\sigma_{N}^{2}$, and $i_{\mathrm{BS}, \mathrm{MT}}^{(1)}, i_{\mathrm{BS}, \mathrm{MT}_{0}}^{(2)}$ are the interference processes affecting $\mathrm{MT}_{0}$, which result from the set of BSs constituting the singleand two-hop transmissions, respectively. They are given by

$$
\begin{aligned}
i_{\mathrm{BS}, \mathrm{MT}_{0}}^{(1)} & =\sum_{\mathrm{BS}_{\mathrm{i}} \in \Phi_{\mathrm{BS}}^{(\mathrm{I}, 1, \text { hop })}} \sqrt{\left(P_{T} / N_{B S}\right) \mathcal{Z}_{\mathrm{BS}_{\mathrm{i}}, \mathrm{MT}_{0}}^{-1}} \mathbf{h}_{\mathrm{BS}_{\mathrm{i}}, \mathrm{MT}_{0}} \\
& \times \mathbf{w}_{\mathrm{BS}_{\mathrm{i}}, \mathrm{MT}_{\mathrm{i}}} s_{i} \mathbf{1}\left(\mathcal{Z}_{\left.\mathrm{BS}_{\mathrm{i}}, \mathrm{MT}_{0}>\mathcal{Z}_{\mathrm{BS}_{0}, \mathrm{MT}_{0}}\right)} \sqrt{K_{T}\left(P_{T} / N_{B S}\right) \mathcal{Z}_{\mathrm{BS}_{\mathrm{j}}, \mathrm{MT}_{0}}^{-1}} \mathbf{h}_{\mathrm{BS}_{\mathrm{j}}, \mathrm{MT}_{0}}\right. \\
& =\sum_{\mathrm{BS}_{\mathrm{j}} \in \Phi_{\mathrm{BS}}^{(\mathrm{I}, 2) \text { hop })}} \sqrt{\times \mathbf{w}_{\mathrm{BS}_{\mathrm{j}}, \mathrm{RN}_{\mathrm{j}}} s_{j} \mathbf{1}}\left(\mathcal{Z}_{\mathrm{BS}_{\mathrm{j}}, \mathrm{MT}_{0}}>\mathcal{Z}_{\mathrm{BS}_{0}, \mathrm{MT}_{0}}\right),
\end{aligned}
$$

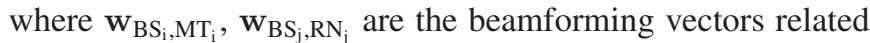
to $\mathrm{BS}_{\mathrm{i}}$ and $\mathrm{BS}_{\mathrm{j}}$, respectively. The indicator functions in (4), $\mathbf{1}\left(\mathcal{Z}_{\mathrm{BS}_{i}, \mathrm{MT}_{0}}>\mathcal{Z}_{\mathrm{BS}_{0}, \mathrm{MT}_{0}}\right)$ and $\mathbf{1}\left(\mathcal{Z}_{\mathrm{BS}_{j}, \mathrm{MT}_{0}}>\mathcal{Z}_{\mathrm{BS}_{0}, \mathrm{MT}_{0}}\right)$ for $\mathrm{BS}_{i} \in \Phi_{\mathrm{BS}}^{(\mathrm{I}, 1 \text { hop })}$ and $\mathrm{BS}_{j} \in \Phi_{\mathrm{BS}}^{(\mathrm{I}, 2 \mathrm{hop})}$, respectively, originate from the association criterion in (1). More specifically, they take into account that the power received from the serving BS is higher than that of every interfering BSs.

Since the serving BSs have perfect CSI of the corresponding channel links towards their served MTs or RNs, we assume that the employed beamforming vectors are designed so that they maximize the received Signal-to-Noise Ratio (SNR). Hence, Maximum Ratio Transmission (MRT) [11] is employed, which means that $\mathbf{w}_{\mathrm{BS}_{0}, \mathrm{MT}_{0}}=\frac{\mathbf{h}_{\mathrm{BS}_{0}, \mathrm{MT}_{0}}^{H}}{\left\|\mathbf{h}_{\mathrm{BS}_{0}, \mathrm{MT}_{0}}\right\|}$. Consequently, the resulting from (3) SINR at $\mathrm{MT}_{0}$, which we denote by $\mathrm{SINR}_{\mathrm{BS}_{0}, \mathrm{MT}_{0}}$, is given by

$$
\mathrm{SINR}_{\mathrm{BS}_{0}, \mathrm{MT}_{0}}=\frac{\left(P_{T} / N_{B S}\right)\left\|\mathbf{h}_{\mathrm{BS}_{0}, \mathrm{MT}_{0}}\right\|^{2} \mathcal{Z}_{\mathrm{BS}_{0}, \mathrm{MT}_{0}}^{-1}}{\sigma_{N}^{2}+I_{\mathrm{BS}, \mathrm{MT}_{0}}^{(1)}+I_{\mathrm{BS}, \mathrm{MT}_{0}}^{(2)}},
$$

where

$$
\begin{aligned}
I_{\mathrm{BS}, \mathrm{MT}_{0}}^{(1)}= & \sum_{\mathrm{BS}_{\mathrm{i}} \in \Phi_{\mathrm{BS}}^{(\mathrm{I}, 1,1 \mathrm{hop})}}\left(P_{T} / N_{B S}\right) \mathcal{Z}_{\mathrm{BS}_{\mathrm{i}}, \mathrm{MT}_{0}}^{-1}\left|\mathbf{h}_{\mathrm{BS}_{\mathrm{i}}, \mathrm{MT}_{0}} \mathbf{w}_{\mathrm{BS}_{\mathrm{i}}, \mathrm{MT}_{\mathrm{i}}}\right|^{2} \\
I_{\mathrm{BS}, \mathrm{MT}_{0}}^{(2)}= & \sum_{\mathrm{BS}_{\mathrm{j}} \in \Phi_{\mathrm{BS}}^{(\mathrm{I}, 2 \mathrm{hop})}} K_{T}\left(\mathcal{Z}_{\mathrm{BS}_{\mathrm{i}}, \mathrm{MT}_{0}}>\mathcal{Z}_{\left.\mathrm{BS}_{0}, \mathrm{MT}_{0}\right)} / N_{B S}\right) \mathcal{Z}_{\mathrm{BS}_{\mathrm{j}}, \mathrm{MT}_{0}}^{-1}\left|\mathbf{h}_{\mathrm{BS}_{\mathrm{j}}, \mathrm{MT}_{0}} \mathbf{w}_{\mathrm{BS}_{\mathrm{j}}, \mathrm{RN}_{\mathrm{j}}}\right|^{2} \\
& \mathbf{1}\left(\mathcal{Z}_{\mathrm{BS}_{\mathrm{j}}, \mathrm{MT}_{0}}>\mathcal{Z}_{\mathrm{BS}_{0}, \mathrm{MT}_{0}}\right) .
\end{aligned}
$$

2) Two-Hop Transmission: In the case that the communication takes place in two hops, $\mathrm{RN}_{0}$ and $\mathrm{BS}_{\mathrm{R} 0}$ are defined in (1). We distinguish two phases of the communication protocol that occur in two subsequent time slots:

First time slot-Communication between $\mathrm{BS}_{\mathrm{R} 0}$ and $\mathrm{RN}_{0}$ : During the first time slot, only the BSs are allowed to transmit. Hence, the received signal at $\mathrm{RN}_{0}$, which we denote by $y_{\mathrm{RN}}$, is given by

$$
\begin{aligned}
y_{\mathrm{RN}_{0}} & =\sqrt{K_{T}\left(P_{T} / N_{B S}\right) Z_{\mathrm{BS}_{\mathrm{R} 0} \mathrm{RN}_{0}}^{-1}} \mathbf{h}_{\mathrm{BS}_{\mathrm{R} 0} \mathrm{RN}_{0}} \mathbf{w}_{\mathrm{BS}_{\mathrm{R} 0} \mathrm{RN}_{0}} \\
& +i_{\mathrm{BS}, \mathrm{RN}_{0}}^{(1)}+i_{\mathrm{BS}, \mathrm{RN}_{0}}^{(2)}+n_{\mathrm{RN}_{0}},
\end{aligned}
$$

where $\mathbf{h}_{\mathrm{BS}_{\mathrm{R} 0}, \mathrm{RN}_{0}} \in \mathbb{C N}^{1 \times N_{B S}}, \mathbf{w}_{\mathrm{BS}_{\mathrm{R} 0}, \mathrm{RN}_{0}} \in \mathbb{C}^{N_{B S} \times 1}$ is the employed beamforming vector at $\mathrm{BS}_{\mathrm{R} 0}, E\left\{n_{\mathrm{RN}_{0}} n_{\mathrm{RN}_{0}}^{*}\right\}=$ $\sigma_{N}^{2}$, and $i_{\mathrm{BS}, \mathrm{RN}_{0}}^{(1)}, i_{\mathrm{BS}, \mathrm{RN}_{0}}^{(2)}$ are the interference processes affecting $\mathrm{RN}_{0}$, which result from the set of BSs constituting the single- and two-hop transmissions, respectively. They are given by

$$
\begin{aligned}
& i_{\mathrm{BS}, \mathrm{RN}_{0}}^{(1)}=\sum_{\mathrm{BS}_{\mathrm{i}} \in \Phi_{\mathrm{BS}}^{(\mathrm{I}, 1 \text { hop })}} \sqrt{\left(P_{T} / N_{B S}\right) \mathcal{Z}_{\mathrm{BS}_{\mathrm{i}}, \mathrm{RN}_{0}}^{-1}} \mathbf{h}_{\mathrm{BS}_{\mathrm{i}}, \mathrm{RN}_{0}} \mathbf{w}_{\mathrm{BS}_{\mathrm{i}}, \mathrm{MT}_{\mathrm{i}}} s_{i} \\
& \mathbf{1}\left(\mathcal{Z}_{\mathrm{BS}_{\mathrm{i}}, \mathrm{RN}_{0}}>\mathcal{Z}_{\mathrm{BS}_{\mathrm{R} 0}, \mathrm{RN}_{0}}\right) s_{i} \mathbf{1}\left(\mathcal{Z}_{\mathrm{BS}_{\mathrm{i}}, \mathrm{MT}_{0}}>\eta_{\text {cell }} \mathcal{Z}_{\mathrm{RN}_{0}, \mathrm{MT}_{0}}\right) \\
& i_{\mathrm{BS}, \mathrm{RN}_{0}}^{(2)}=\sum_{\mathrm{BS}_{\mathrm{j}} \in \Phi_{\mathrm{BS}}^{(\mathrm{I}, 2 \mathrm{hop})}} \sqrt{K_{T}\left(P_{T} / N_{B S}\right) \mathcal{Z}_{\mathrm{BS}_{\mathrm{j}}, \mathrm{RN}_{0}}^{-1}} \mathbf{h}_{\mathrm{BS}_{\mathrm{j}}, \mathrm{MT}_{0}} \mathbf{w}_{\mathrm{BS}_{\mathrm{j}}, \mathrm{RN}_{\mathrm{j}}} s_{j} \\
& \mathbf{1}\left(\mathcal{Z}_{\mathrm{BS}_{\mathrm{j}}, \mathrm{RN}_{0}}>\mathcal{Z}_{\mathrm{BS}_{\mathrm{R} 0}, \mathrm{RN}_{0}}\right) \mathbf{1}\left(\mathcal{Z}_{\mathrm{BS}_{\mathrm{j}}, \mathrm{MT}_{0}}>\eta_{\text {cell }} \mathcal{Z}_{\mathrm{RN}_{0}, \mathrm{MT}_{0}}\right),
\end{aligned}
$$

where the indicator functions of (8) originate from (1) and (2). According to the MRT criterion, it holds that $\mathbf{w}_{\mathrm{BS}_{\mathrm{R} 0}, \mathrm{RN}_{0}}=$ $\frac{\mathbf{h}_{\mathrm{BS}_{\mathrm{R} 0}, \mathrm{RN}_{0}}^{H}}{\left\|\mathbf{h}_{\mathrm{BS}_{\mathrm{R} 0}, \mathrm{RN}_{0}}\right\|}$. Hence, the resulting from (7) SINR at $\mathrm{RN}_{0}$, which we denote by $\mathrm{SINR}_{\mathrm{BS}_{\mathrm{R} 0}, \mathrm{RN}_{0}}$, is given by

$$
\mathrm{SINR}_{\mathrm{BS}_{\mathrm{R} 0}, \mathrm{RN}_{0}}=\frac{K_{T}\left(P_{T} / N_{B S}\right)\left\|\mathbf{h}_{\mathrm{BS}_{\mathrm{R} 0}, \mathrm{RN}_{0}}\right\|^{2} \mathcal{Z}_{\mathrm{BS}_{\mathrm{R} 0}, \mathrm{RN}_{0}}^{-1}}{\sigma_{N}^{2}+I_{\mathrm{BS}, \mathrm{RN}_{0}}^{(1)}+I_{\mathrm{BS}, \mathrm{RN}_{0}}^{(2)}},
$$

where

$$
\begin{aligned}
& I_{\mathrm{BS}, \mathrm{RN}}(1)=\sum_{\mathrm{BS}_{\mathrm{i}} \in \Phi_{\mathrm{BS}}^{(\mathrm{I}, \text { Ihop })}}\left(P_{T} / N_{B S}\right) \mathcal{Z}_{\mathrm{BS}_{\mathrm{i}}, \mathrm{RN}_{0}}^{-1}\left|\mathbf{h}_{\mathrm{BS}_{\mathrm{i}}, \mathrm{RN}_{0}} \mathbf{w}_{\mathrm{BS}_{\mathrm{i}}, \mathrm{MT}_{\mathrm{i}}}\right|^{2} \\
& \mathbf{1}\left(\mathcal{Z}_{\mathrm{BS}_{\mathrm{i}}, \mathrm{RN}_{0}}>\mathcal{Z}_{\mathrm{BS}_{\mathrm{R} 0}, \mathrm{RN}_{0}}\right) \mathbf{1}\left(\mathcal{Z}_{\mathrm{BS}_{\mathrm{i}}, \mathrm{MT}_{0}}>\eta_{\text {cell }} \mathcal{Z}_{\mathrm{RN}_{0}, \mathrm{MT}_{0}}\right) \\
& I_{\mathrm{BS}, \mathrm{RN}_{0}}^{(2)}= \sum_{\mathrm{BS}_{\mathrm{j}} \in \Phi_{\mathrm{BS}}^{(\mathrm{I}, 2 \mathrm{hop})}} K_{T}\left(P_{T} / N_{B S}\right) \mathcal{Z}_{\mathrm{BS}_{\mathrm{j}}, \mathrm{RN}_{0}}^{-1}\left|\mathbf{h}_{\mathrm{BS}_{\mathrm{j}}, \mathrm{RN}_{0}} \mathbf{w}_{\mathrm{BS}_{\mathrm{j}}, \mathrm{RN}_{\mathrm{j}}}\right|^{2} \\
& \mathbf{1}\left(\mathcal{Z}_{\mathrm{BS}_{\mathrm{j}}, \mathrm{RN}_{0}}>\mathcal{Z}_{\mathrm{BS}_{\mathrm{R} 0}, \mathrm{RN}_{0}}\right) \mathbf{1}\left(\mathcal{Z}_{\mathrm{BS}_{\mathrm{j}}, \mathrm{MT}_{0}}>\eta_{\text {cell }} \mathcal{Z}_{\mathrm{RN}_{0}, \mathrm{MT}_{0}}\right) .
\end{aligned}
$$

Second time slot-Communication between $\mathrm{RN}_{0}$ and $\mathrm{MT}_{0}$ : During the second time slot, only the RNs are allowed to 
transmit. Hence, the received signal at $\mathrm{RN}_{0}$, which we denote by $y_{\mathrm{MT}_{0}}$, is given by

$$
\begin{aligned}
y_{\mathrm{MT}_{0}}^{(2)} & =\sqrt{\left(1-K_{T}\right)\left(P_{T} / N_{R S}\right) \mathcal{Z}_{\mathrm{RN}_{0}, \mathrm{MT}_{0}}^{-1}} \mathbf{h}_{\mathrm{RN}_{0}, \mathrm{MT}_{0}} \\
& \times \mathbf{w}_{\mathrm{RN}_{0}, \mathrm{MT}_{0}} s^{\prime}+i_{\mathrm{RN}, \mathrm{MT}_{0}}^{(2)}+n_{\mathrm{MT}_{0}}^{(2)},
\end{aligned}
$$

where $s^{\prime}$ is the resulting modulated signal after the detection of $s$ at $\mathrm{RN}_{0}, \mathbf{h}_{\mathrm{RN}_{0}, \mathrm{MT}_{0}} \in \mathbb{C N}^{1 \times N_{R S}}, \mathbf{w}_{\mathrm{RN}_{0}, \mathrm{MT}_{0}} \in$ $\mathbb{C}^{N_{R S} \times 1}$ is the employed beamforming vector at $\mathrm{RN}_{0}$, $E\left\{n_{\mathrm{MT}_{0}}^{(2)} n_{\mathrm{MT}_{0}}^{(2) *}\right\}=\sigma_{N}^{2}$, and $i_{\mathrm{RN}, \mathrm{MT}_{0}}^{(2)}$ is the interference process affecting $\mathrm{MT}_{0}$, which results from the set of interfering RSs. It is given by

$$
\begin{aligned}
& i_{\mathrm{RN}, \mathrm{MT}_{0}}^{(2)}=\sum_{\mathrm{RN}_{k} \in \Phi_{\mathrm{RN}}^{(\mathrm{I})}} \sqrt{\left(1-K_{T}\right)\left(P_{T} / N_{R S}\right) \mathcal{Z}_{\mathrm{RN}_{\mathrm{k}}, \mathrm{MT}_{0}}^{-1}} \mathbf{h}_{\mathrm{RN}_{\mathrm{k}}, \mathrm{MT}_{0}} \\
& \operatorname{card}\left\{\Phi_{\mathrm{RN}}^{(\mathrm{I})}\right\}=\operatorname{card}\left\{\Phi_{\mathrm{BS}}^{(\mathrm{I}, 2 \mathrm{hop})}\right\} \\
& \quad \times \mathbf{w}_{\mathrm{RN}_{\mathrm{k}}, \mathrm{MT}_{0} s_{k} \mathbf{1}\left(\mathcal{Z}_{\mathrm{RN}_{k}, \mathrm{MT}_{0}}>\mathcal{Z}_{\left.\mathrm{RN}_{0}, \mathrm{MT}_{0}\right)}\right.}
\end{aligned}
$$

where the indicator function in (12) results from the association criterion (1). According to the MRT criterion, it holds that $\mathbf{W}_{\mathrm{RN}_{0}, \mathrm{MT}_{0}}=\frac{\mathbf{h}_{\mathrm{RN}_{0}, \mathrm{MT}_{0}}^{H}}{\left\|\mathbf{h}_{\mathrm{RN}_{0}, \mathrm{MT}_{0}}\right\|}$. Hence, the resulting from (11) SINR at $\mathrm{MT}_{0}$, which we denote by $\mathrm{SINR}_{\mathrm{RN}_{0}, \mathrm{MT}_{0}}$, is given by

$$
\operatorname{SINR}_{\mathrm{RN}_{0}, \mathrm{MT}_{0}}=\frac{\left(1-K_{T}\right)\left(P_{T} / N_{R S}\right)\left\|\mathbf{h}_{\mathrm{RN}_{0}, \mathrm{MT}_{0}}\right\|^{2} \mathcal{Z}_{\mathrm{RN}_{0}, \mathrm{MT}_{0}}^{-1}}{\sigma_{N}^{2}+I_{\mathrm{RN}, \mathrm{MT}_{0}}^{(2)}},
$$

where

$$
\begin{aligned}
& I_{\mathrm{RN}^{\prime} \mathrm{MT}_{0}}^{(2)}=\sum_{\mathrm{RN}_{k} \in \Phi_{\mathrm{RN}}^{(\mathrm{I})}}\left(1-K_{T}\right)\left(P_{T} / N_{R S}\right)\left|\mathbf{h}_{\mathrm{RN}_{\mathrm{k}}, \mathrm{MT}_{0}} \mathrm{w}_{\mathrm{RN}_{\mathrm{k}}, \mathrm{MT}_{0}}\right|^{2} \\
& \operatorname{card}\left\{\Phi_{\mathrm{RN}}^{(\mathrm{I})}\right\}=\operatorname{card}\left\{\Phi_{\mathrm{BS}}^{(\mathrm{I}, 2 \mathrm{hop})}\right\} \\
& \times \mathcal{Z}_{\mathrm{RN}_{k}, \mathrm{MT}_{0}}^{-1} \mathbf{1}\left(\mathcal{Z}_{\mathrm{RN}_{k}, \mathrm{MT}_{0}}>\mathcal{Z}_{\mathrm{RN}_{0}, \mathrm{MT}_{0}}\right) .
\end{aligned}
$$

\section{B. Examined Metrics}

Two performance metrics are considered in this work that are subsequently defined: 1) coverage probability and 2) average rate.

1) Coverage Probability: As coverage probability, which we denote by, $\mathrm{P}_{\text {cov }}(\mathrm{T})$ we define the probability that the received SINR of either the single- or the two-hop transmission is greater than a threshold $\mathrm{T}$. It is given by

$$
\begin{aligned}
\mathrm{P}_{\mathrm{cov}}(\mathrm{T}) & =\mathbb{E}_{\mathcal{Z}_{\mathrm{BS}_{0}, \mathrm{MT}_{0}}}\left\{\mathrm{P}_{\mathrm{cov}}^{(1 \text { hop })}\left(\mathrm{T} ; \mathcal{Z}_{\mathrm{BS}_{0}, \mathrm{MT}_{0}}\right)\right\} \\
& +\mathbb{E}_{\mathcal{Z}_{\mathrm{RN}_{0}, \mathrm{MT}_{0}}}\left\{\mathrm{P}_{\mathrm{cov}}^{(2 \mathrm{hop})}\left(\mathrm{T} ; \mathcal{Z}_{\mathrm{RN}_{0}, \mathrm{MT}_{0}}\right)\right\},
\end{aligned}
$$

where $\mathrm{P}_{\mathrm{cov}}^{(1 \mathrm{hop})}(\cdot ; \cdot)$ and $\mathrm{P}_{\mathrm{cov}}^{(2 \mathrm{hop})}(\cdot ; \cdot)$ are the coverage probabilities corresponding to one- and two-hop links, respectively, defined in [7, Eq.(10)]. The conditioning upon $\mathcal{Z}_{\mathrm{BS}_{0}, \mathrm{MT}_{0}}$ and $\mathcal{Z}_{\mathrm{RN}_{0}, \mathrm{MT}_{0}}$ originates from (2).

2) Average Rate: The average rate, $R_{\text {average, which is the }}$ expectation of the instantaneous rate, is defined as

$$
\begin{aligned}
& \mathrm{R}_{\text {average }}=\mathbb{E}_{\mathcal{Z}_{\mathrm{BS}_{0}, \mathrm{MT}_{0}}}\left\{\mathrm{R}_{\text {average }}^{\text {(1hop) }}\left(\mathcal{Z}_{\mathrm{BS}_{0}, \mathrm{MT}_{0}}\right)\right\} \\
& +\mathbb{E}_{\mathcal{Z}_{\mathrm{RN}_{0}, \mathrm{MT}_{0}}}\left\{\mathrm{R}_{\text {average }}^{(\text {2hop })}\left(\mathcal{Z}_{\mathrm{RN}_{0}, \mathrm{MT}_{0}}\right)\right\},
\end{aligned}
$$

where $\mathrm{R}_{\text {average }}^{(1 \text { hop })}\left(\mathcal{Z}_{\mathrm{BS}_{0}, \mathrm{MT}_{0}}\right)$ and $\mathrm{R}_{\text {average }}^{(2 \mathrm{hop})}\left(\mathcal{Z}_{\mathrm{BS}_{0}, \mathrm{MT}_{0}}\right)$ are defined in [7, Eq.(12)]

\section{Performance Analysis}

In this section, we derive expressions for the examined performance metrics of coverage probability and average rate. Towards this, i) At first, we present the analytical expressions of the required distributions to derive these expressions. ii) We consider useful geometrical approximations.

\section{A. Preliminaries}

It is evident from Section III-B that the derivation of the analytical expressions of the coverage probability and average rate requires the computation of the following quantities: i) The distribution of $\mathcal{Z}_{X_{0}, Y_{0}}=l\left(r_{X_{0}, Y_{0}}\right) / S_{X_{0}, Y_{0}}$, which we denote by $F_{\mathcal{Z}_{X_{0}, Y_{0}}}(\cdot)$ and it is given by [7, Eq. (31)], ii) The conditional probabilities that $\mathrm{MT}_{0}$ is served via a one-hop and a two-hop link, i.e.

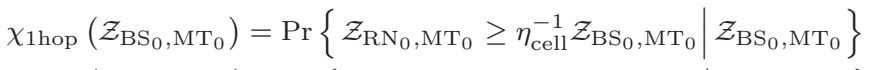

$\chi_{\text {2hop }}\left(\mathcal{Z}_{\mathrm{RN}_{0}, \mathrm{MT}_{0}}\right)=\operatorname{Pr}\left\{\mathcal{Z}_{\mathrm{BS}_{0}, \mathrm{MT}_{0}}>\eta_{\text {cell }} \mathcal{Z}_{\mathrm{RN}_{0}, \mathrm{MT}_{0}} \mid \mathcal{Z}_{\mathrm{RN}_{0}, \mathrm{MT}_{0}}\right\}$,

which are given by [7, Eq. (34)] and iii) The probabilities that $\mathrm{MT}_{0}$ is served via a one- and a two-hop link, i.e., $\chi_{1 \text { hop }}$ and $\chi_{2 \mathrm{hop}}$ with $\chi_{1 \mathrm{hop}}$ given by $\left[7\right.$, Eq. (35)] and $\chi_{2 \mathrm{hop}}=1-\chi_{1 \mathrm{hop}}$.

\section{B. Considered Approximations}

As in [7], we consider the following approximations regarding the indicator functions of $I_{\mathrm{BS}, \mathrm{RN}_{0}}^{(1)}$ and $I_{\mathrm{BS}, \mathrm{RN}_{0}}^{(2)}$ :

$$
\begin{aligned}
I_{\mathrm{BS}, \mathrm{RN}}(1) & \approx \sum_{\mathrm{BS}_{\mathrm{i}} \in \Phi_{\mathrm{BS}}^{(\mathrm{I}, \text { hhop })}}\left(P_{T} / N_{B S}\right) \mathcal{Z}_{\mathrm{BS}_{\mathrm{i}}, \mathrm{RN}_{0}}^{-1}\left|\mathbf{h}_{\mathrm{BS}_{\mathrm{i}}, \mathrm{RN}_{0}} \mathbf{w}_{\mathrm{BS}_{\mathrm{i}}, \mathrm{MT}_{\mathrm{i}}}\right|^{2} \\
& \mathbf{1}\left(\mathcal{Z}_{\mathrm{BS}_{\mathrm{i}}, \mathrm{RN}_{0}>}>\mathcal{Z}_{\left.\mathrm{BS}_{\mathrm{R} 0}, \mathrm{RN}_{0}\right)}\right) \\
I_{\mathrm{BS}, \mathrm{RN}_{0}}^{(2)} \approx & \sum_{\mathrm{BS}_{\mathrm{j}} \in \Phi_{\mathrm{BS}}^{(\mathrm{I}, 2 \mathrm{hop})}} K_{T}\left(P_{T} / N_{B S}\right) \mathcal{Z}_{\mathrm{BS}_{\mathrm{j}}, \mathrm{RN}_{0}}^{-1}\left|\mathbf{h}_{\mathrm{BS}_{\mathrm{j}}, \mathrm{RN}_{0}} \mathbf{w}_{\mathrm{BS}_{\mathrm{j}}, \mathrm{RN}_{\mathrm{j}}}\right|^{2} \\
& \mathbf{1}\left(\mathcal{Z}_{\mathrm{BS}_{\mathrm{j}}, \mathrm{RN}_{0}}>\mathcal{Z}_{\mathrm{BS}_{\mathrm{R} 0}, \mathrm{RN}_{0}}\right),
\end{aligned}
$$

which are valid approximations regarding the realistic case for a wide range of relay densities, as it is shown in the numerical results of [7].

\section{Coverage Probability}

Proposition 1: The coverage probability defined in (14) is approximated as

where

$$
\mathrm{P}_{\mathrm{Cov}}(\mathrm{T}) \approx \mathcal{J}_{\mathrm{BS}, \mathrm{MT}}(\mathrm{T})+\mathcal{J}_{\mathrm{BS}, \mathrm{RN}}(\mathrm{T}) \mathcal{J}_{\mathrm{RN}, \mathrm{MT}}(\mathrm{T}),
$$

$\mathcal{J}_{\mathrm{BS}, \mathrm{MT}}(\mathrm{T})=\pi \lambda_{\mathrm{BS}} \mathrm{Y}_{\mathrm{BS}, \mathrm{MT}} \int_{0}^{\infty} \exp \left(-\pi \lambda_{\mathrm{BS}} \mathrm{Y}_{\mathrm{BS}, \mathrm{MT}} \mathrm{y}\right)$

$\times \exp \left(-\frac{\pi \lambda_{\mathrm{RN}} \mathrm{Y}_{\mathrm{RN}, \mathrm{MT}}}{\eta_{\text {cell }}^{2 / \beta_{\mathrm{RN}, \mathrm{MT}}}} \mathrm{y}^{\frac{\beta_{\mathrm{BS}, \mathrm{MT}}}{\beta_{\mathrm{RN}, \mathrm{MT}}}}\right) \times \sum_{n=0}^{N_{B S}-1} \frac{1}{n !}\left(-\kappa_{0} \mathrm{y}^{\frac{\beta_{\mathrm{BS}, \mathrm{MT}}}{2}}\right)^{n}$

$\times \exp \left(-\frac{\kappa_{0} \mathrm{y}^{\frac{\beta_{\mathrm{BS}, \mathrm{MT}}}{2}} \sigma_{N}^{2} \mathrm{~T}}{P_{T} / N_{B S}}\right) \times \sum_{k=0}^{n}\left(\begin{array}{c}n \\ k\end{array}\right)\left(-\frac{\sigma_{N}^{2} \mathrm{~T}}{P_{T} / N_{B S}}\right)^{n-k}$

$\times p_{\mathrm{BS}, \mathrm{MT}}^{(k)}(\mathrm{y}) \mathrm{dy}$. 


$$
\begin{aligned}
& \mathcal{J}_{\mathrm{BS}, \mathrm{RN}}(\mathrm{T})=\pi \lambda_{\mathrm{BS}} \mathrm{Y}_{\mathrm{BS}, \mathrm{RN}} \int_{0}^{\infty} \exp \left(-\pi \lambda_{\mathrm{BS}} \mathrm{Y}_{\mathrm{BS}, \mathrm{RN}} \mathrm{y}\right) \\
& \times \sum_{n=0}^{N_{B S}-1} \frac{1}{n !}\left(-\kappa_{0} \mathrm{y} \frac{\beta_{\mathrm{BS}, \mathrm{RN}}}{2}\right)^{n} \times \exp \left(-\frac{\kappa_{0} \frac{\beta_{\mathrm{BS}, \mathrm{RN}}}{2} \sigma_{N}^{2} \mathrm{~T}}{P_{T} / N_{R S}}\right) \\
& \times \sum_{k=0}^{n}\left(\begin{array}{c}
n \\
k
\end{array}\right)\left(-\frac{\sigma_{N}^{2} \mathrm{~T}}{P_{T} / N_{B S}}\right)^{n-k} p_{\mathrm{BS}, \mathrm{RN}}^{(k)}(\mathrm{y}) \mathrm{dy} .
\end{aligned}
$$

$$
\begin{aligned}
& \mathcal{J}_{\mathrm{RN}, \mathrm{MT}}(\mathrm{T})=\pi \lambda_{\mathrm{RN}} \mathrm{Y}_{\mathrm{RN}, \mathrm{MT}} \int_{0}^{\infty} \exp \left(-\pi \lambda_{\mathrm{RN}} \mathrm{Y}_{\mathrm{RN}, \mathrm{MT}} \mathrm{y}\right) \\
& \times \exp \left(-\frac{\pi \lambda_{\mathrm{BS}} \mathrm{Y}_{\mathrm{BS}, \mathrm{MT}}}{\eta_{\mathrm{cell}}^{-2 / \beta_{\mathrm{BS}, \mathrm{MT}}}} \mathrm{y}^{\frac{\beta_{\mathrm{RN}, \mathrm{MT}}}{\beta_{\mathrm{BS}, \mathrm{MT}}}}\right) \times \sum_{n=0}^{N_{R S}-1} \frac{1}{n !}\left(\kappa_{0} \mathrm{y}^{\frac{\beta_{\mathrm{RN}, \mathrm{MT}}}{2}}\right)^{n} \\
& \times \exp \left(-\frac{\kappa_{0} \mathrm{y} \frac{\beta_{\mathrm{RN}, \mathrm{MT}}}{2} \sigma_{N}^{2} \mathrm{~T}}{P_{T} / N_{R S}}\right) \times \sum_{k=0}^{n}\left(\begin{array}{c}
n \\
k
\end{array}\right)\left(-\frac{\sigma_{N}^{2} \mathrm{~T}}{P_{T} / N_{R S}}\right)^{n-k} \\
& \times p_{\mathrm{RN}, \mathrm{MT}}^{(k)}(\mathrm{y}) \mathrm{dy},
\end{aligned}
$$

where

$$
p_{\mathrm{X}, \mathrm{Y}}^{(k)}(\mathrm{y})=\left\{\begin{aligned}
\exp \left(g_{\mathrm{X}, \mathrm{Y}}(\mathrm{y})\right) & , \\
\exp \left(g_{\mathrm{X}, \mathrm{Y}}(\mathrm{y})\right) & \times \sum \frac{k !}{p_{1} ! \ldots p_{k} !}\left(\frac{g_{\mathrm{X}, \mathrm{Y}}^{(1)}(\mathrm{y})}{1 !}\right)^{p_{1}} \times \ldots \\
& \times \ldots\left(\frac{g_{\mathrm{X}, \mathrm{Y}}^{(k)}(\mathrm{y})}{k !}\right)^{p_{k}}, k \geq 1
\end{aligned}\right.
$$

if $k \geq 1$ and where $\mathrm{X}, \mathrm{Y}=\{(\mathrm{BS}, \mathrm{MT}),(\mathrm{BS}, \mathrm{RN}),(\mathrm{RN}, \mathrm{MT})\}, p_{1}, \ldots p_{k}$ are given by the non-negative integer solutions of the equation $p_{1}+2 p_{2}+$ $\ldots k p_{k}=k, g_{\mathrm{X}, \mathrm{Y}}(\mathrm{y})=B_{\mathrm{X}, \mathrm{Y}} \mathrm{y}$, and

$$
g_{\mathrm{X}, \mathrm{Y}}^{(k)}(\mathrm{y})=B_{\mathrm{X}, \mathrm{Y}} \prod_{m=1}^{k}\left(\frac{2}{\beta_{\mathrm{X}, \mathrm{Y}}}-(m-1)\right)\left(\kappa_{0}\right)^{-m_{\mathrm{y}}-m \frac{\beta_{\mathrm{X}, \mathrm{Y}}}{2}+1},
$$

with

$$
\begin{aligned}
B_{\mathrm{BS}, \mathrm{MT}} & =\pi \lambda_{\mathrm{BS}}^{(\mathrm{I}, \mathrm{hop})} \Upsilon_{\mathrm{BS}, \mathrm{MT}} \Psi_{\mathrm{BS}, \mathrm{MT}}(-x) \\
& +\pi \lambda_{\mathrm{BS}}^{(\mathrm{I}, 2 \mathrm{hop})} \Upsilon_{\mathrm{BS}, \mathrm{MT}} \Psi_{\mathrm{BS}, \mathrm{MT}}\left(-K_{T} x\right) \\
B_{\mathrm{BS}, \mathrm{RN}} & =\pi \lambda_{\mathrm{BS}}^{(\mathrm{I}, 1 \mathrm{hop})} \Upsilon_{\mathrm{BS}, \mathrm{RN}} \Psi_{\mathrm{BS}, \mathrm{RN}}\left(-x / K_{T}\right) \\
& +\pi \lambda_{\mathrm{BS}}^{(\mathrm{I}, 2 \mathrm{hop})} \Upsilon_{\mathrm{BS}, \mathrm{RN}} \Psi_{\mathrm{BS}, \mathrm{RN}}(-x) \\
B_{\mathrm{RN}, \mathrm{MT}} & =\pi \lambda_{\mathrm{BS}}^{(\mathrm{I}, 2 \mathrm{hop})} \Upsilon_{\mathrm{RN}, \mathrm{MT}} \Psi_{\mathrm{RN}, \mathrm{MT}}(-x),
\end{aligned}
$$

where $\Psi_{\mathrm{BS}, \mathrm{MT}}(x), \Psi_{\mathrm{BS}, \mathrm{RN}}(x)$, and $\Psi_{\mathrm{RN}, \mathrm{MT}}(x)$ are given by [7, Eq. (22)].

Proof: See APPENDIX.

\section{Average Rate}

Proposition 2: The average rate defined in (15) is approximated as

$$
\begin{aligned}
\mathrm{R}_{\text {average }} & =\frac{\mathrm{B}_{\mathrm{W}}}{\ln (2)} \int_{0}^{+\infty} \mathcal{J}_{\mathrm{BS}, \mathrm{MT}}(z) \frac{d z}{z+1} \\
& +\frac{1}{2} \frac{\mathrm{B}_{\mathrm{W}}}{\ln (2)} \int_{0}^{+\infty} \mathcal{J}_{\mathrm{BS}, \mathrm{RN}}(z) \mathcal{J}_{\mathrm{RN}, \mathrm{MT}}(z) \frac{d z}{z+1}
\end{aligned}
$$

Proof: See [7, Section IV-B].

\section{Numerical Results}

Regarding the simulation parameters, we consider $P_{T}=45$ $\mathrm{dBm}, K_{T}=0.5, N_{B S}=N_{R S}=N_{T}, \mathcal{F}_{\mathrm{dB}}=10 \mathrm{~dB}, f_{c}=2$ $\mathrm{GHz}$ is the carrier frequency, $\mu_{X, Y}=0 \mathrm{~dB}, \sigma_{X, Y}=4 \mathrm{~dB}$, $\Omega_{X, Y}=1, \mathrm{~B}_{\mathrm{W}}=180 \mathrm{kHz}, \mathrm{N}_{\mathrm{RB}}=50, \lambda_{\mathrm{BS}}=1 /\left(\pi R_{\mathrm{cell}}^{2}\right)$, where $R_{\text {cell }}=200 \mathrm{~m}$ is the average radius of a cell, and $\lambda_{\mathrm{RN}}=3 \lambda_{\mathrm{BS}}$. It is worth mentioning that $\mathrm{B}_{\mathrm{W}}=180 \mathrm{kHz}$ is the transmission bandwidth of a RB in the LTE-A standard. $\lambda_{\mathrm{MT}}^{(\mathrm{RB})}=(1 / 5) \lambda_{\mathrm{BS}}$ is the density of the MTs, which are served at the same RB as $\mathrm{MT}_{0}$. Hence, it is the density of the interfering BSs. Regarding the Monte Carlo simulations, the same principles as in [7, Section V] are followed.

Fig. 1 illustrates the coverage probability and average rate for $\beta_{\mathrm{BS}, \mathrm{MT}}=4.5$ and $\beta_{\mathrm{BS}, \mathrm{RN}}=\beta_{\mathrm{RN}, \mathrm{MT}}=3$, different number of available antennas at the BSs and the RNs, and the cases of no-RNs and of the value of $\mathcal{B}_{\mathrm{BS}}$ that maximizes the coverage probability. This value was obtained from the analytical model through linear search. The intuition behind this lies on the fact that in realistic scenarios RNs are expected to be needed to assist the communication between BSs and MT for high $\beta_{\mathrm{BS}, \mathrm{MT}}$. Considering this, RNs are expected to be deployed in a way that the BS-RN and RN-MT links are stronger than the BS-MT links, which means that RNs are likely to be deployed on rooftops [15]. As we observe from Fig. 1, there is a close match between the analytical and simulation results. The same conclusions can be drawn for $\beta_{\mathrm{BS}, \mathrm{MT}}=4.5$, where we observe significant coverage gains for $N_{T}=100$. However, the average rate in this case is substantially reduced due to the half-duplex constrained of the RNs if the optimization of $\mathcal{B}_{\mathrm{BS}}$ is solely based on the maximization of the coverage probability.

\section{CONCLUSIONS}

In this work, we have extended the analysis of [7] in relay-aided cellular networks by considering MRT-based and interference unaware beamforming through the use of multiple antennas at the BSs and RNs. Analytical expressions are provided regarding the achieved coverage probability and average rate, which show a close match with respect to Monte Carlo simulations. The results reveal interesting trends regarding the usefulness of RNs conditioned the number of antennas at the BSs and RNs. In particular, if the number of antennas at the BSs and RNs is relatively high, significant coverage gains can be achieved with respect to the no-RNs case by placing the RNs in a way that the path-loss exponent of the BS-RN and RN-MT links is relatively low. However, due to the half-duplex constrained of the RNs no gain in terms of average rate is observed. In fact, if the objective is only the maximization of the coverage probability, it leads to an average-rate reduction with respect to the no-RNs case. This tradeoff motivates the study of the full-duplex RNs case in our future work.

\section{ACKNOWLEDGMENTS}

This work was supported in part by the Generalitat de Catalunya and the CellFive Project under Grants 2014-SGR1551 and TEC2014-60130-P, respectively. 


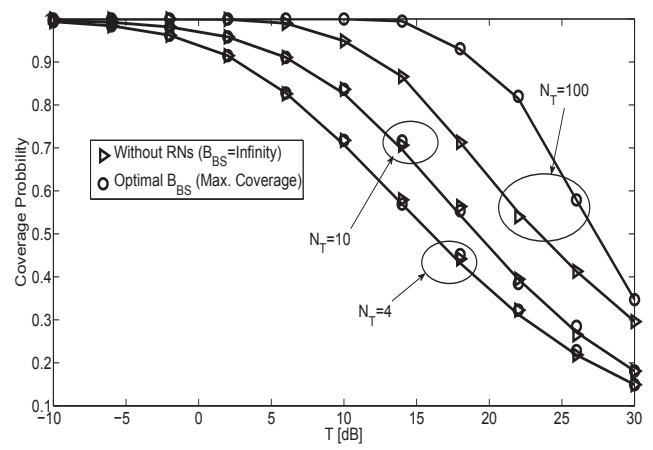

(a) Coverage probability.

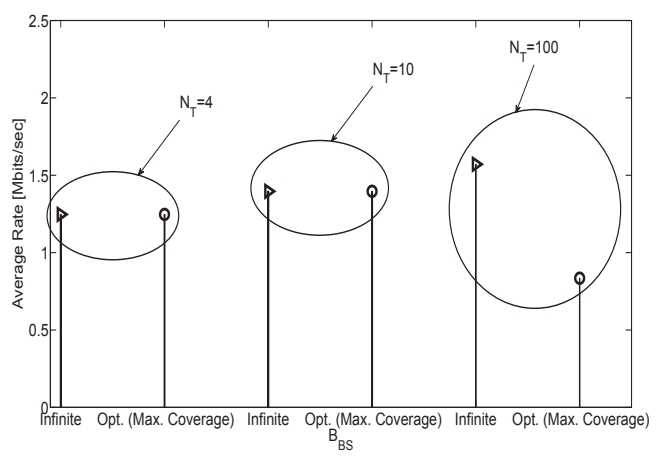

(b) Average rate.

Fig. 1: Coverage probability and average rate for $\beta_{\mathrm{BS}, \mathrm{MT}}=$ 4.5 and $\beta_{\mathrm{BS}, \mathrm{RN}}=\beta_{\mathrm{RN}, \mathrm{MT}}=3$. Solid lines illustrate the analytical model, whereas markers illustrate Monte Carlo simulations.

\section{APPENDIX}

Proof of Proposition 1 : For $\mathcal{J}_{\mathrm{BS}, \mathrm{MT}}(\mathrm{T})$, it holds that $\mathcal{J}_{\mathrm{BS}, \mathrm{MT}}(\mathrm{T})=\mathbb{E}_{\mathcal{Z}_{\mathrm{BS}_{0}, \mathrm{MT}}}\left\{\mathrm{P}_{\text {cov }}^{(1 \text { hop })}\left(\mathrm{T} ; \mathcal{Z}_{\mathrm{BS}_{0}, \mathrm{MT}}\right)\right\}$. By considering [7, Eq. (10)] for the expression of $\mathrm{P}_{\text {cov }}^{(1 \text { hop })}\left(\mathrm{T} ; \mathcal{Z}_{\mathrm{BS}_{0}, \mathrm{MT}_{0}}\right)$, we have

$\operatorname{Pr}\left\{\mathrm{SINR}_{\mathrm{BS}_{0}, \mathrm{MT}_{0}}>\mathrm{T} \mid \mathcal{Z}_{\mathrm{BS}_{0}, \mathrm{MT}_{0}}\right\}$

$\stackrel{(a)}{=} \operatorname{Pr}\left\{\left\|\mathbf{h}_{\mathrm{BS}_{0}, \mathrm{MT}_{0}}\right\|^{2}>V_{\mathcal{Z}_{\mathrm{BS}_{0}, \mathrm{MT}_{0}}} \mid \mathcal{Z}_{\mathrm{BS}_{0}, \mathrm{MT}_{0}}\right\}$

$\stackrel{(b)}{=} E_{(\cdot) \backslash \mathcal{Z}_{\mathrm{BS}_{0}, \mathrm{MT}_{0}}}\left\{\sum_{n=0}^{N_{B S}-1} \frac{1}{n !}\left(V_{\mathcal{Z}_{\mathrm{BS}_{0}, \mathrm{MT}_{0}}}\right)^{n} \exp \left(-V_{\mathcal{Z}_{\mathrm{BS}_{0}, \mathrm{MT}_{0}}}\right)\right\}$

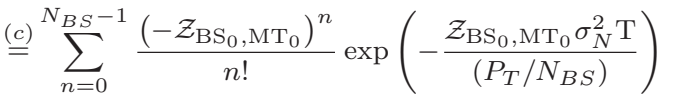

$\times \sum_{k=0}^{n}\left(\begin{array}{c}n \\ k\end{array}\right)\left(-\frac{\sigma_{N}^{2} \mathrm{~T}}{\left(P_{T} / N_{B S}\right)}\right) \frac{d^{k}}{d\left(\mathcal{Z}_{\left.\mathrm{BS}_{0}, \mathrm{MT}_{0}\right)^{k}}\right.} \mathscr{L}_{G_{\mathrm{BS}, \mathrm{MT}}}\left(\mathcal{Z}_{\mathrm{BS}_{0}, \mathrm{MT}_{0}}\right)$

where $V_{\mathcal{Z}_{\mathrm{BS}_{0}, \mathrm{MT}_{0}}}=\frac{Z_{\mathrm{BS}_{0}, \mathrm{MT}_{0} \mathrm{~T}}}{\left(P_{T} / N_{B S}\right)}\left(\sigma_{N}^{2}+I_{\mathrm{BS}, \mathrm{MT}_{0}}^{(1)}+I_{\mathrm{BS}, \mathrm{MT}_{0}}^{(2)}\right)$,

$\mathscr{L}_{G_{\mathrm{BS}, \mathrm{MT}}}\left(\mathcal{Z}_{\mathrm{BS}_{0}, \mathrm{MT}_{0}}\right)=E_{(\cdot) \backslash Z_{\mathrm{BS}_{0}, \mathrm{MT}_{0}}}\left\{\exp \left(-G_{\mathrm{BS}, \mathrm{MT}}\left(Z_{\mathrm{BS}_{0}, \mathrm{MT}_{0}}\right)\right)\right\}$

with $G_{B S, M T}=Z_{\mathrm{BS}_{0}, \mathrm{MT}_{0}} \frac{\mathrm{T}}{\left(P_{T} / N_{B S}\right)}\left(I_{\mathrm{BS}, \mathrm{MT}_{0}}^{(1)}+I_{\mathrm{BS}, \mathrm{MT}_{0}}^{(2)}\right)$,

follows from (5), (b) follows due to the fact that $\left\|\mathbf{h}_{\mathrm{BS}_{0}, \mathrm{MT}_{0}}\right\|^{2}$ is a chi-squared random variable with $N_{B S}$ degrees of freedom [11], and, finally, $(c)$ follows from the properties of the Laplace transform together with the product rule of the higher derivatives of the product of two functions. $\mathscr{L}_{G_{B S, M T}}\left(\mathcal{Z}_{B S_{0}, M T_{0}}\right)$ can be analytically derived by considering [7, Eq. (38), Eq. (40)], the fact that

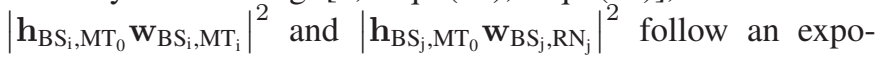
nential distribution [12], and, finally, [13, Lemma 1], which leads to the analytical expression of $\mathcal{J}_{\mathrm{BS}, \mathrm{MT}}(\mathrm{T})$ in (19). By taking into account that $\mathcal{J}_{\mathrm{BS}, \mathrm{RN}}(\mathrm{T}) \mathcal{J}_{\mathrm{RN}, \mathrm{MT}}(\mathrm{T})=$ $\mathbb{E}_{\mathcal{Z}_{\mathrm{RN}_{0}, \mathrm{MT}_{0}}}\left\{\mathrm{P}_{\text {cov }}^{(2 \mathrm{hop})}\left(\mathrm{T} ; \mathcal{Z}_{\mathrm{RN}_{0}, \mathrm{MT}_{0}}\right)\right\}$, that the received instantaneous SINRs at $\mathrm{RN}_{0}$ and $\mathrm{MT}_{0}$ are independent due to different sets of interferers affecting these nodes [7], and by following the same steps as in the derivation of $\mathcal{J}_{\mathrm{BS}, \mathrm{MT}}(\mathrm{T})$, $\mathcal{J}_{\mathrm{BS}, \mathrm{RN}}(\mathrm{T})$ and $\mathcal{J}_{\mathrm{RN}, \mathrm{MT}}(\mathrm{T})$ are obtained, which concludes the proof of Proposition 1.

\section{REFERENCES}

[1] IEEE 802.16 Relay Task Group, Multi-hop relay system evaluation methodology, IEEE 802.16j-6/013r3, Feb. 2007.

[2] S. W. Peters, A. Y. Panah, K. T. Truong, and R. W. Heath Jr. "Relay architectures for 3GPP LTE-advanced", EURASIP J. Wireless Commun and Networking, vol. 2009, 14 pages, July 2009.

[3] H. A. Suraweera, H. K. Garg, and A. Nallanathan, "Performance analysis of two hop amplify-and-forward systems with interference at the relay", IEEE Commun. Lett., vol. 14, no. 8, pp. 692-694, Aug. 2010.

[4] F. Al-Qahtani, T. Duong, C. Zhong, K. Qaraqe, and H. Alnuweiri, "Performance analysis of dual-hop AF systems with interference in Nakagami- $m$ fading channels", IEEE Sig. Proc. Lett., vol. 18, no. 8, pp. 454-457, Aug. 2011.

[5] X. Liang, S. Jin, W. Wang, X. Gao, and K.-K. Wong, "Outage probability of amplify-and-forward two-way relay interference-limited systems", IEEE Trans. Veh. Technol., vol. 61, no. 6, pp. 2221-2231, June 2013.

[6] S. S. Ikki and S. Aissa, "Performance analysis of two-way amplifyand- forward relaying in the presence of co-channel interference", IEEE Trans. Commun., vol. 60, no. 4, pp. 933``939, Apr. 2012.

[7] W. Lu and M. Di Renzo, "Stochastic Geometry Modeling and SystemLevel Analysis/Optimization of Relay-Aided Downlink Cellular Networks", IEEE Trans. Commun., vol. PP, no. PP, Sep. 2015.

[8] F. Baccelli and B. Blaszczyszyn, Stochastic Geometry and Wireless Networks, Part I: Theory, Now Publishers, Sep. 2009.

[9] H.-S. Jo, Y. J. Sang, P. Xia, and J. G. Andrews, "Heterogeneous cellular networks with flexible cell association: A comprehensive downlink SINR analysis", IEEE Trans. Wireless Commun., vol. 11, no. 10, pp. 34843495, Oct. 2012.

[10] M. Di Renzo, A. Guidotti, and G. E. Corazza, "Average rate of downlink heterogeneous cellular networks over generalized fading channels - A stochastic geometry approach", IEEE Trans. Commun., vol. 61, no. 7, pp. 3050-3071, July 2013.

[11] T. K. Y. Lo, "Maximum ratio transmission", IEEE Trans. Commun., vol. 47, no. 10, Aug. 2002.

[12] A. Shah and A. M. Haimovich, "Performance analysis of maximal ratio combining and comparison with optimum combining for mobile radio communications with cochannel interference", IEEE Trans. Veh. Technology, vol. 49, no. 4, July 2000.

[13] X. Zhou, J. Guo, S. Durrani, and I. Krikidis "Performance of Maximum Ratio Transmission in Ad Hoc Networks with SWIPT", IEEE Wirel. Commun. Letters, vol. PP, no. PP, July 2015.

[14] T.L. Marzetta, "Noncooperative Cellular Wireless with Unlimited Numbers of Base Station Antennas", IEEE Trans. Wirel. Commun., vol. 9, no. 11 , July 2010.

[15] M. Di Renzo and Wei Lu, "End-to-End Error Probability and Diversity Analysis of AF-based Dual-Hop Cooperative Relaying in a Poisson Field of Interferers at the Destination", IEEE Trans. Commun., vol. 14, no. 1, Jan. 2015. 